\title{
Replacement of biologic by molecular techniques in diagnostic virology: Thirty years after the advent of PCR technology-do we still need conventional methods?
}

\author{
H. W. Doerr
}

Received: 13 July 2013/ Accepted: 10 August 2013/Published online: 21 August 2013

(c) Springer-Verlag Berlin Heidelberg 2013

\begin{abstract}
About 150 years ago, Louis Pasteur discovered that transmissible diseases and epidemics are caused by microbes or even subcellular infectious agents, later on called viruses.

The first methods to detect and to identify those agents were microscopy, animal experiments or voluntary self infections of scientists. Pasteur, Robert Koch and others developed a lot of methods to cultivate, to isolate and to identify bacteria in special bouillons, agar dishes, methods, which have been continuously improved and optimized up to date. In virology, a first breakthrough in the diagnostic technology of infectious diseases was the development of embryonated chicken eggs as the smallest system of animal experiments in the thirties of the last century and the advent of cell cultures in the subsequent decade.
\end{abstract}

For the next three decades, cell culture technology was the basis of diagnostic virology in terms of three aspects:

- By the use of cell culture technology, many viruses were isolated and propagated in large amounts.

- This opened up the closer research on viruses and supported the development of antivirals and vaccines.

- Cell culture technology provides antigens for the antibody detection and for immunization of animals to produce specific antibodies for antigen detection (serology).

But there were three disadvantages:

- Biologic tests (e.g., determination of cytopathic effects of viruses) or serologic tests with a biologic test signal

H. W. Doerr $(\square)$

Institute of Medical Virology, Johann Wolfgang Goethe

University, Frankfurt/M, Germany

e-mail: H.W.Doerr@em.uni-frankfurt.de (cell agglutination, complement fixation, suppression of $\mathrm{CPE}$ ) cannot be easily standardized in terms of test reproducibility and quantification.

- Biologic tests do not rapidly provide test results, since virus propagation needs time, although considerable improvement is gained by detection of early antigens in virus-infected cells.

- Serological tests are rapid. However, antigen detection suffers from limited sensitivity (though signal amplification, e.g., peroxidase-antiperoxidase test). Apart from immunopathogenic diseases, antibody formation and subsequently determination is a late diagnostic event.

Tests of molecular biologic diagnostics are methods to determine genomic nucleic acid of the infectious agents or of transcribed mRNA.

Since 1970, those tests were developed using the avidity between the strands in genomic DNA for diagnostic approaches similar to the avidity between antigen and antibody. The first tests were developed to detect nonculturable viruses either in blood or other fluid samples (e.g., dot blot hybridization of hepatitis B virus DNA) in organ tissue samples (e.g., in situ hybridization of oncogenic DNA viruses). Apart from hybridization, viral DNA or RNA electrophoresis was applied: Electrophore typing of segmented virus RNA genome (e.g., rotavirus) and restriction fragment length polymorphism (RFLP) of DNA viruses (and of cDNA from RNA viruses) proved of value as simple tools of molecular epidemiology and tracing infection chains.

Those tests were widely distributed. However, their sensitivity was not satisfying.

A real breakthrough in diagnostics of infectious agents, in particular of viruses, was achieved when in 1983 Kary Mullis developed the technique of serially repeated DNA 
hybridizations as polymerase chain reaction (PCR) to rapidly amplify selected DNA sequences to large amounts. The method bases upon and expands the technique of DNA sequencing, as introduced by Frederick Sanger 10 years earlier. After reverse transcription, genomic sequences of RNA viruses or mRNA transcripts can be amplified by cDNA PCR, too. In this way, virus cultivation and isolation can be replaced by amplification and detection of selected viral genes. In the last 30 years, numerous modifications have been developed and applied in all fields of biochemical and microbiologic research [1]. Among them, the real time PCR has achieved highest relevance in diagnostic virology. In this method, the reaction kinetics is faithfully determined during the hybridization cycles by release of defined signal-emitting gene probes. As a consequence, the relative amount of viral genomes can be calculated. In blood (plasma) samples, the viraemia can be quantified. The advantages of PCR compared to the conventional cell culture-based methods of virus detection are as follows:

- Detection and counting of non- or not easily culturable viruses (e.g., HBV, HCV, HIV)

- Rapid pathognomonic test results in special specimens, e.g., CSF for the diagnosis of neurotropic virus infections

- Reduced problems of specimen sampling and transport in comparison to virus isolation procedure

- Superior turnaround time (results are achieved in hours instead of days) without significant loss of sensitivity or specificity compared to conventional virus isolation procedures

- Superior specificity compared to other rapid tests (antigen, EM)

- Quantification of viraemia as a marker of pathogenicity and therapy control (e.g., HIV, HCV, CMV)

- Superior management in hygiene and epidemiology:

- Genotyping by rapid nucleic acid sequencing,

- Exclusion of infectious risks

A main advantage is virus detection and quantification in the pathologic material, e.g., in bronchial lavages of patients suffering from pneumonia. On the other side, sometimes the etiologic relevance is questionable in terms of what is the hen (infectious etiology)? What is the egg (secondary reactivation)? [2].
A second break-through in viral diagnostics was achieved by the advent of automated methods of DNA sequencing which have replaced all former methods to identify and characterize a virus and track infection chains [3]. Modern methods of "next generation sequencing" have revolutionized genetics and provide information on viral and microbial pathogenicity [4].

This was of particular importance in the management of HIV diseases since the advent of multiple anti-retroviral drugs. However, new technologies produce new problems, too.

- Problems related to high sensitivity: "more results than symptoms!" (definition of cut-off lines is needed in terms of clinical relevance)

- Analysis of therapy resistance by viral genotyping has to be based on data bases and requires computer analysis, while conventional phenotyping provides reliable results in individual cases, but is timeconsuming.

- The determination of disinfectant or antiviral drug activity and of neutralizing/protecting antibodies cannot do without biologic technologies to assess reduction in infectivity

In summary, molecular methods have replaced biologic methods in many aspects of routine diagnostics of infectious diseases. However, in special cases, conventional methods remain useful or even essential (cell culture technology, hemagglutination, and electron microscopy).

\section{References}

1. Doerr HW, Cinatl J (2012) Recent publications in medical microbiology and immunology: a retrospective. Med Microbiol Immunol 201:1-5

2. Friedrichs I, Bingold T, Keppler OT, Pullmann B, Reinheimer C, Berger A (2013) Detection of herpes virus EBV DNA in the lower respiratory tract of ICU patients: a marker of infection of the lower respiratory tract? Med. Microbiol Immunol 202: ePub ahead of print

3. Stürmer M, Doerr HW (2009) Gürtler L (2009) Human immunodeficiency virus: 25 years of diagnostic and therapeutic strategies and their impact on hepatitis B and C virus. Med Microbiol Immunol 198(3):147-155

4. Chiu CY (2013) Viral pathogen discovery. Curr Opin Microbiol (ePub ahead of print) 